\section{Pan drug-resistant Serratia marcescens: An emerging threat}

To the Editor: The five most frequently reported organisms isolated from blood cultures worldwide are Klebsiella pneumoniae, Escherichia coli, Staphylococcus aureus, Pseudomonas aeruginosa and Enterococcus species. ${ }^{[1,2]}$ The emergence of carbapenem resistance has seriously compromised treatment options for organisms such as K. pneumoniae, E. coli and other Enterobacteriaceae such as Enterobacter species and Serratia marcescens. Colistin and tigecycline are last-resort therapeutic options for these carbapenem-resistant Enterobacteriaceae. ${ }^{[3]}$

We have observed an increase in the number of carbapenemresistant $S$. marcescens isolates in blood cultures in our laboratory in the KwaZulu-Natal (KZN) region, South Africa, which is particularly worrisome, given that Serratia species are inherently resistant to colistin.

During 2017, S. marcescens was the 6th most common isolate in blood cultures. Of the 572 Enterobacteriaceae isolated from blood cultures, S. marcescens was the $3 \mathrm{rd}$ most common after E. coli and K. pneumoniae, accounting for $11 \%$ (63/572) of isolates. Ten S. marcescens isolates were carbapenem resistant; 7 of these were also resistant to tigecycline, thus making them pan drug resistant. All the carbapenemresistant S. marcescens isolates carried the New Delhi metallo-betalactamase 1 (NDM-1) gene. Unlike other reports of S. marcescens bacteraemia, these isolates were from several different hospitals across KZN and were not linked to an outbreak. ${ }^{[4,5]}$
The emergence of pan drug-resistant $S$. marcescens in blood cultures in this region creates a challenge for the management of patients admitted to hospitals and intensive care units. Implementation of antimicrobial stewardship and strict infection control practices is imperative to prevent further dissemination of this formidable pathogen.

\section{K Moodley, A K C Peer, C N Govind}

Department of Microbiology, Lancet Laboratories, Durban; and

Antimicrobial Research Unit, College of Health Sciences,

University of KwaZulu-Natal, Durban, South Africa

krishnee.moodley@lancet.co.za

1. Johnson AP. Surveillance of antibiotic resistance. Philos Trans R Soc Lond B Biol Sc 2015;370(1670):20140080. https://doi.org/10.1098/rstb.2014.008

2. Laupland KB. Incidence of bloodstream infection: A review of population-based studies. Clin Microbiol Infect 2013;19(6):492-500. https://doi.org/10.1111/1469-0691.12144

3. Alhashem F, Tiren-Verbeet NL, Alp E, Doganayt M. Treatment of sepsis: What is the antibiotic choice in bacteremia due to carbapenem resistant Enterobacteriaceae? World J Clin Cases 2017;5(8):324-332 https://doi.org/10.12998/wjcc.v5.i8.324

4. Iosifidis E, Farmaki E, Nedelkopoulou N, et al. Outbreak of bloodstream infections because of Serratia marcescens in a pediatric department. Am J Infect Control 2011;40(1):11-15. https://doi.org/10.1016/j. ajic.2011.03.020

5. Pan A, Dolcetti L, Barosi C, et al. An outbreak of Serratia marcescens bloodstream infections associated with misuse of drug vials in a surgical ward. Infect Control Hosp Epidemiol 2006;27(1):79-82. https:// doi.org/10.1086/500250

S Afr Med J 2018;108(4):251. DOI:10.7196/SAMJ.2018.v108i4.13159 